\title{
Building of Multi-Tier Web Server Access Control Design and Research
}

\author{
${ }^{1}$ PAN Dan, ${ }^{2}$ GAN Hong \\ ${ }^{1}$ Central South University ,Changsha,China \\ ${ }^{12}$ Guangzhou City Construction College, Guangzhou, China \\ pandanvip@163.com gbxing@163.com
}

\section{KeyWords: Multi-Tiered Web Server; Admission Control; Session; Fuzzy Control}

\begin{abstract}
The general multi-tiered admission control strategies restrict the system throughput for two reasons. Firstly there is no consideration on the dependency between every tier, and secondly the strategies have the delay characteristic. In this paper, a 2-level fuzzy admission control algorithm is proposed to solve this problem. The first level named load controller judges the load situation of each tier by the resource consumption and requests delay. The second level named admission controller decides the admission rate of the current session by probability statistics. The experiments show that the algorithm can overcome the limitation of general admission control strategies and improve the system throughput.
\end{abstract}

\section{Introduction}

With the explosive growth of Web traffic, the Web server systems often face pressure overload, the serious influence the performance of the server system, its performance is usually: consumption of system resource utilization is too high and even all the system resources, a significant reduction in the throughput and the response time of the request or response cannot be obtained many requests. But the Web server performance and scale impossible unlimited upgrade to meet some sudden traffic, it is undoubtedly an economic waste. Therefore, on the Web server of access control, also known as overload protection, become the essential means to prevent Web server system overload. Previous monolayer on the Web server access control to static Web pages to simulate Web load, but with the increasing scale and complexity of Web applications, more modern Web application relies on the dynamic content ${ }^{[1]}$. Request to and different from the static, dynamic page cannot cache or replacement and the user load are more difficult to predict. Therefore, the study on access control in multilayer on the Web server has become a hot issue nowadays.

\section{General Multi-Storey Web Server Access Control}

Multiple servers, each server can provide certain function for the server, and at the same time can make full use of the back of the server resources, to better respond to user request ${ }^{[2]}{ }^{[3]}$. Modern Web applications in general can be divided into three layers, as shown in figure 1: the Web server's performance as a three layers structure layer; The middle layer application server as processing center of all logic and data processing layer, the main processing user dynamic requests; Background database is the place where the user to store data information, can be put in one or more host, to provide information collection, search, product management and other services.

The basic idea of the access control of the multi - layer Web server is ${ }^{[11]}$ : the access rate of different session types and different levels of server resource usage are proportional. In general, the database server is sensitive to the Browsing (Mix), and the application server is sensitive to the Ordering Shopping (Mix), while the Web server is sensitive to the order type session (Mix). To browse the type of session as an example, according to the TPC WIRT Web time (interaction response), the type of the Web server layer, application server layer and database server layer resource requirements of $20 \%, 30$ and $50 \%$.

In the admission control of Web server, in each sampling period, the monitor is used to predict the resource usage of the current server according to the measurement value of the current server. This is due to different server types, and the Web request types are not the same. Through statistical analysis of the characteristics of load request, the access control of multi server system can realize the balance of different levels of resources and effectively increase the system throughput. 
Literature ${ }^{[4]}$,In the electronic commerce Web site, the access control of different requests is realized in a session. The ${ }^{[5]}$ is implemented by user's purchase record as the classification standard. The ${ }^{[6]}$ server is implemented in the multi layer Web server system. The access control algorithm is proposed. Literature ${ }^{[8] 9]}$, using the method of queuing theory to analyze the application of multi-layer Web. Among them, ${ }^{[8]}$ The application of the queue model to determine the number of servers assigned to each layer of Web application, and ${ }^{[9]}$ The application server layer on the establishment of the optimal model based on queuing theory.

All of the above studies are based on the multi-layer Web server, and realize the access control, but there are some defects in the ${ }^{[11]}$ :

Only the load of each layer is predicted separately, without taking into account the level of coordination among the operations;

Access control policy is implemented only at the edge of the sampling time, with a certain delay. If the access policy for the moment is to reject all sessions, even if the resource of a layer of the server is idle at the next time, the policy continues until the next sampling period.

In view of the above problems, in on the basis of the research, literature ${ }^{[10]}$ to Betsey network as a reasoning tool design coordination based on multilayer web access session control (Cossack, coordinated Session-based admission control with statistical learning for using Internet Applications). In this paper, from the point of view of control theory, the function of the Bayesian network access control is accomplished by designing the 2 stage fuzzy controller, and it can not only refuse or accept a session, but also improve the system throughput.

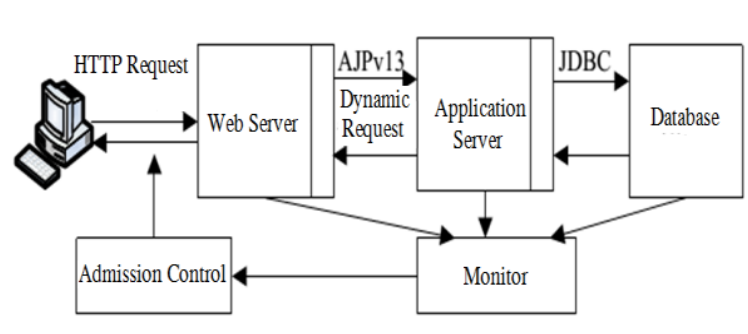

Fig.1. Three Layer Web Server Architecture

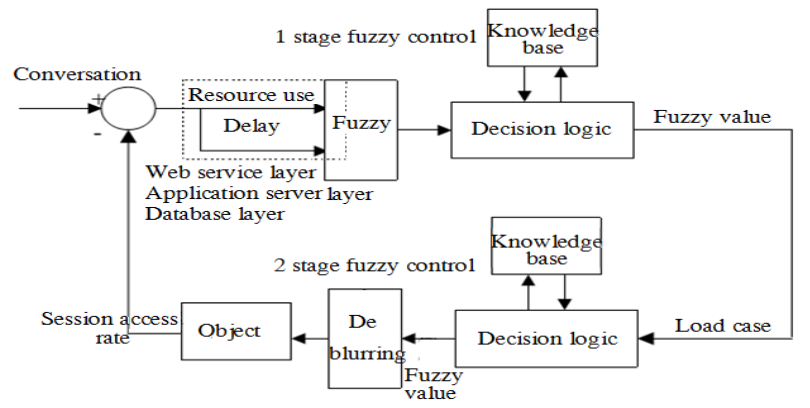

Fig.2. Three-tier Web server 2 levels of access control

\section{Fuzzy Controller Design.}

As shown in figure 2, this paper USES two level fuzzy control to accomplish multiple Web server access control: level 1 as the load controller, input object for the current session of arrival, variable for each server resource utilization and processing delay, output objects for each server load (fuzzy value); Level 2 for access controller, input object as the output of the controller at the next higher level, namely load at all levels, output object is the current level of sensitive session access rate of the P. The access rate compared with the random number $\mathrm{x}$, the random number is from the $\mathrm{U}(0,1)$ of the distribution of a sample, if $\mathrm{x}<=\mathrm{P}$, accept the session; whereas a refusal. If the request at this time is already accept service session, then the request acceptable probability is $100 \%$.

\subsection{Load the Fuzzy Controller}

Fuzzy control, fuzzy controller for level 1 load on each server using respectively, aim for each server load. Due to the level of output as the subordinate controller input, so don't need to make to fuzzy results. Because the server is handled at all levels are consistent, therefore only represented by the Web server layer.

Define variables: The level for the two-dimensional fuzzy controller, fuzzy controller which consists of two input variables: delay Delay Resource resource utilization and processing. Output variables for Load load conditions.

Blur: For the convenience of narrative, linguistic variables for Resourcew, there are five language variable values: \{use US use UB is small, small, normal use NM, large use OS, large use OB \}; Delay EC for language variables, there are five language variable values: $\{$ small small NB, NS, normal NM, larger PS, big PB $\}$, the basic theory of domain were taken as the [0,100], [0, | Delayw|Max], quantitative factors, 4/100,4/|Delayw|Max. Output variables $\mathrm{U}$ for Load, there are five language variable values: \{under load bigger UB, under load smaller US, normal load NM, overload smaller OS, large overload OB \}, the scaling factor is 4/100.

Knowledge Base: According to expert experience and knowledge, get the following control rules table. 
Fuzzy Reasoning: When the Web server layer of resource usage and delay to obey Gaussian membership functions, and load obey the triangle membership function, fuzzy rules surface figure is shown in figure 3 .

\begin{tabular}{|c|c|c|}
\hline $\begin{array}{l}\text { Linguistic } \\
\text { variables }\end{array}$ & $\begin{array}{c}\text { Basic theory } \\
\text { of domain }\end{array}$ & Language value and value center \\
\hline $\mathrm{E}$ & {$[0,100]$} & $\begin{array}{l}\{\mathrm{UB}, \mathrm{US}, \mathrm{NM}, \mathrm{OS}, \mathrm{OB}\} \\
\{0,20,50,75,100\} 5 \text { 档 }\end{array}$ \\
\hline $\mathrm{EC}$ & {$\left[0, \mid\right.$ Delay $\left.\left._{w}\right|_{\max }\right]$} & $\begin{array}{c}\left\{0, \mid \text { Delay }\left._{\mathrm{w}}\right|_{\max } / 4,, \mid \text { Delay }\left._{\mathrm{w}}\right|_{\max } / 2\right. \\
\left.3, \mid \text { Delay }\left._{\mathrm{w}}\right|_{\max } / 4,, \mid \text { Delay }\left._{\mathrm{w}}\right|_{\max }\right\}\end{array}$ \\
\hline $\mathrm{U}$ & {$[0,100]$} & $\begin{array}{l}\{\mathrm{UB}, \mathrm{US}, \mathrm{NM}, \mathrm{OS}, \mathrm{OB}\} \\
\{0,20,50,75,100\} 5 \text { 档 }\end{array}$ \\
\hline
\end{tabular}

Table1. Load controller variable fuzzy table.

\begin{tabular}{|c|c|c|c|c|c|c|c|}
\hline E & $\mathrm{U}$ & E & UB & US & UM & OS & $\mathrm{OB}$ \\
\hline & NB & & UB & UB & US & US & NM \\
\hline & NS & & UB & US & US & $\mathrm{NM}$ & OS \\
\hline & $\mathrm{NM}$ & & US & US & $\mathrm{NM}$ & OS & $\mathrm{OB}$ \\
\hline & PS & & US & NM & OS & OB & $\mathrm{OB}$ \\
\hline & PB & & NM & OS & OB & OB & $\mathrm{OB}$ \\
\hline
\end{tabular}

Table2. Load controller control rules table.

3.2 Access to the fuzzy controller: Define variables: access to the input variable of fuzzy controller $\mathrm{E}$ as the output of the controller at the next higher level, namely load situation of the current layer, there are five language variable values: \{ under load bigger UB, under load smaller US, normal load NM, overload smaller OS, large overload OB $\}$. Domain for $[0,100]$ the basic theory, theory of domain are $1,2,3,4,5$, quantification factors were 4100 .

Output variables $U$ for access to the session layer sensitive type, there are five language variable values: $\{R A$ completely refusal to RB, accepts the edge AS, accept AB, and fully accept AA\}. Domain for $[0,100]$ the basic theory, theory of domain are 1,2,3,4,5, and the scaling factor is 4100 .

Table3. Access variable fuzzy controller table

\begin{tabular}{|c|c|c|}
\hline $\begin{array}{c}\text { Linguistic } \\
\text { variables }\end{array}$ & $\begin{array}{c}\text { Basic theory } \\
\text { of domain }\end{array}$ & Language value and value center \\
\hline $\mathrm{E}$ & {$[0,100]$} & $\begin{array}{l}\{\mathrm{UB}, \mathrm{US}, \mathrm{NM}, \mathrm{OS}, \mathrm{OB}\} \\
\{0,20,50,75,100\} 5 \text { 档 }\end{array}$ \\
\hline $\mathrm{U}$ & {$[0,100]$} & $\begin{array}{l}\{\mathrm{UB}, \mathrm{US}, \mathrm{NM}, \mathrm{OS}, \mathrm{OB}\} \\
\{0,20,50,75,100\} 5 \text { 档 }\end{array}$ \\
\hline
\end{tabular}

- Knowledge Base: The one dimensional fuzzy controller rules table is as follows:

\section{- Fuzzy Reasoning}

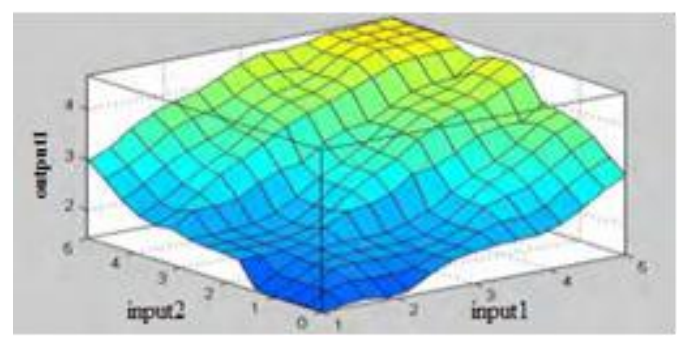

Fig. 3 Fuzzy rules surface of load controller
Table4. Access control rules table

\begin{tabular}{cccccc}
\hline $\mathrm{E}$ & $\mathrm{UB}$ & $\mathrm{US}$ & $\mathrm{NM}$ & $\mathrm{OS}$ & $\mathrm{OB}$ \\
\hline $\mathrm{U}$ & $\mathrm{AA}$ & $\mathrm{AB}$ & $\mathrm{AS}$ & $\mathrm{RB}$ & $\mathrm{RA}$ \\
\hline
\end{tabular}

\section{Experimental verification}

4.1 Experimental configuration: Test - bed by four 100 Mbpsethernet PC running Windows platform, including 1 as a Web server, the server software for Apache2.0.53, 100 concurrent service thread; One run Tomcat6.0.13 as the second layer of the application server, the connection pool to allow the maximum number of connections is set to 50; 1 running MySQL as the back-end database.

Another 1 PC running TPC-WJAVA application as a load generator. TPC-W is TPC committee (Transaction Processing Council) published in 2000 a benchmark e-commerce applications, but does not provide the source code. So modify the TPC released by the University of Wisconsin - Madison PHARM lab - WJAVA application (ver1.5) that is compatible with the experimental platform, and run the program on the PC as a load generator. Customer requests based on session (session), load intensity is determined by the number of concurrent simulation 
browser. Every session, which request the client think time between averages of 0.7 seconds, a obey exponential distribution, the database is composed of 10 tables, contains 10000 terms and 288000 customers.

4.2 Result of the experiment and analysis: To verify the effect of different access control algorithm in multi-storey Web server, more general access control, Bayesian network access control ${ }^{[9]}$ and Browsing session type fuzzy control in load weight for mix: Shopping mix: Ordering mix=1:1:1,case system throughput, as shown in fig.5. Session request arrival rate between 10 and s to $100 \mathrm{~s}$, multi-storey Web server system under different

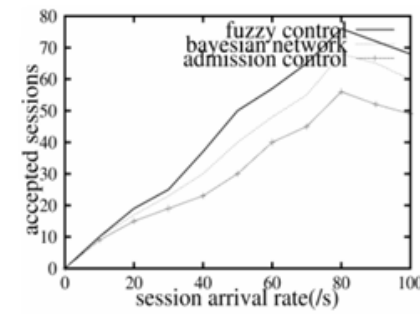

(a) Accept the session number

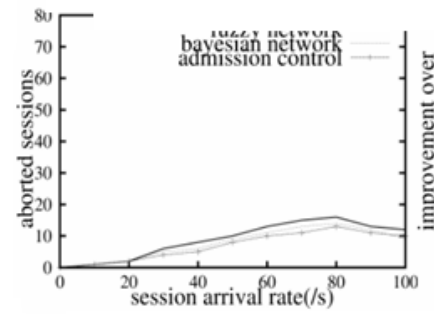

(d) Number of Sessions in the Suspension

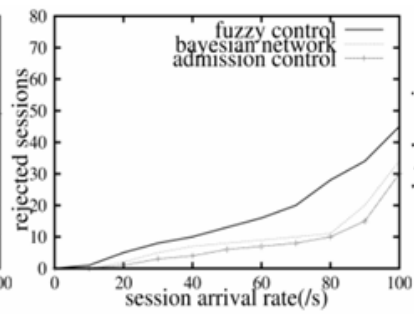

(b) Number of sessions in the refuse

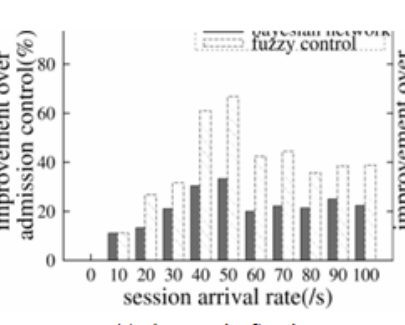

(e) Accept the Session to

Optimize Percentage

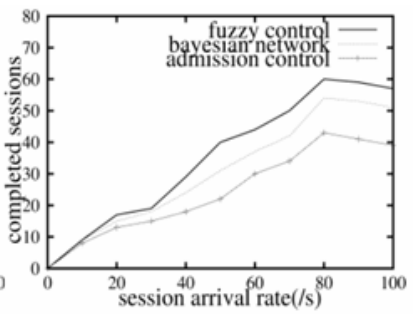

(c) Accept the full number of sessions

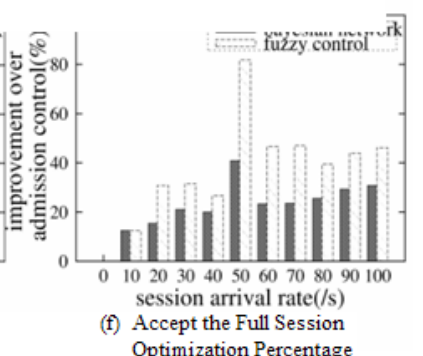

Accept the Full Session
Optimization Percentage

access algorithm of number of sessions, rejection, number of sessions, accept the complete number and suspended a number of the session.

Fig.5. Session throughput under different access algorithms when the load ratio 1:1:1

The experimental results show that, compared with general access control, Bayesian network access control, fuzzy control can effectively improve the multi-storey Web system accept session number and complete the number of sessions. Select request arrival rate of $80 \mathrm{a} / \mathrm{s}$, the system is in a state of saturation, for example, in the access control as a general reference, Bayesian network access control and fuzzy access control on accepting the session number respectively increased by $21.4 \%$ and $35.7 \%$; In a full session number, increased by $25.6 \%$ and $39.5 \%$. Analysis of the possible reasons, there are:

Bayesian networks and fuzzy access strategy to overcome the defect of the general access strategy (such as stated in the introduction), can adapt to different sessions for different levels of server resource consumption, improve the system throughput;

Access control and Bayesian network control. Probabilistic inference as the latter is the core of the control algorithm; the threshold setting depends entirely on the designer's personal experience. And on the fuzzy control can fully inherit the advantage of Bayesian network, which can be considered the interaction relationship between each server levels, and in each session access to judge arrived; On the other hand, differs from that of Bayesian network, fuzzy control can be integrated a number of expert opinion, the division of fuzzy reasoning fine-grained better.

Fig.6 for Browsing load proportion mix: Shopping mix: Ordering mix=5, departure session throughput under different access algorithm. The curve shape similar to figure 5, but the overall session throughput fell slightly. This is mainly because of proportion in the session; the load can be a more balanced and unified was consumed by each server. Session ratio imbalance will lead to some layer to bear more loads, allowing more session is rejected or aborted. But no matter what kind of load, fuzzy access control is reflected its advantages in terms of throughput. 


\section{Conclusion}

Based on the analysis of the general multi-storey Web server system access control algorithm, it is concluded that the limitation of its existence. Literature ${ }^{[10]}$ through the way of Bayesian network to probability reasoning good solve the problem, this article is from the perspective of control theory, a two level fuzzy control is put forward, it good inherits the advantages of the former on the one hand, on the other hand, more powerful expert knowledge reasoning according to oneself, to achieve the better allocation of resources, and its superiority in throughput is verified by experiment.
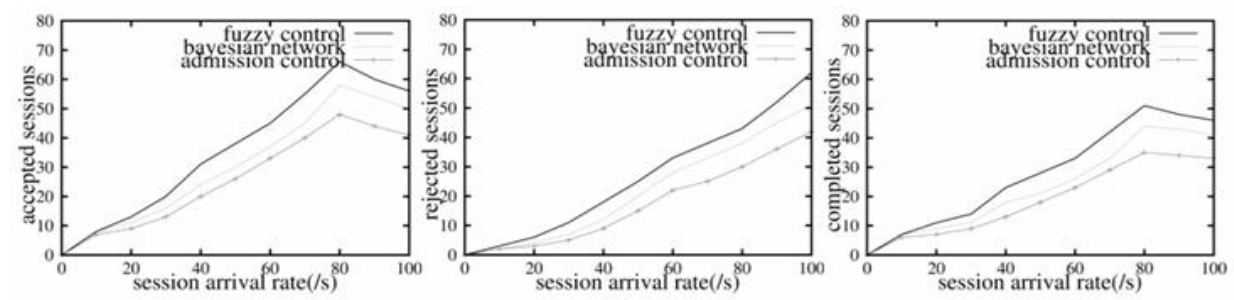

(a) Accept the session number

(b) Number of sessions in the refuse

(c) Accept the full number of sessions
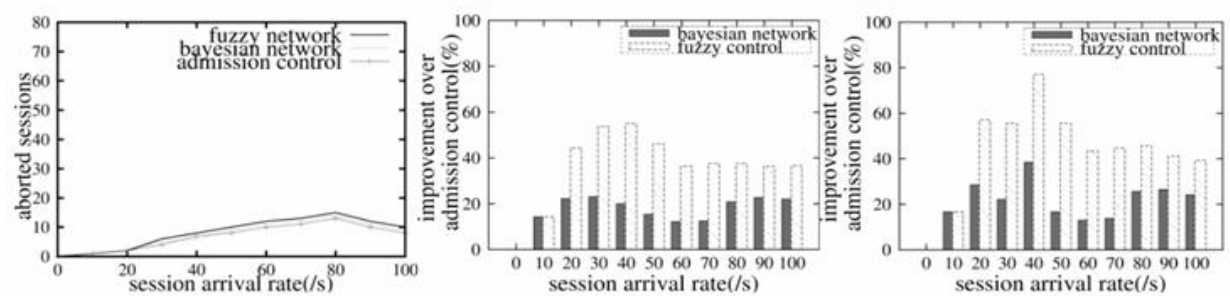

(d) Number of Sessions in

(e) Accept the Session to Optimize Percentage

(f) Accept the Full Session Optimization Percentage

Fig.6. Session throughput under different access algorithms when the load ratio 1:2:3

\section{CONFLICT OF INTEREST}

The authors confirm that this article content has no conflicts of interest.

\section{ACKNOWLEDGMENT}

This work was supported by the Guangdong natural fund team research project, China (no.S2012030006242), National Natural Science Foundation of China (no.61272067).

\section{Reference}

[1]Rio J,Up C.Online measurement of the capacity of multi-tier websites using hardware performance counters[C].In Proc. IEEE Int'l Conf. on Distributed Computing Systems,2008.

[2]Lama P, Zhou X. Efficient server provisioning with delay guarantee on multi-tier clusters[C].In Procaine Int'1 Workshop on Quality of Service (Winos),2009.

[3]Uppsala S, Zhou X. Cossack: coordinated session admission control for multi-tier Internet applications[C].In Proc. IEEE Int'l Conf. on Computer Communications and Networks,2009.

[4]Zhou X, Weir J, Up C Z. Resource allocation for session-based two-dimensional service differentiation on e-commerce servers [J]. IEEE Trans. on Parallel and Distributed Systems, 2006,17(8):838-850.

[5]Yuen C, Wang H N. Profit-aware admission control for overload protection in E-competence Web sites[C]. In Procaine Int'1 Workshop on Quality of Service (Winos),2007.

[6]Same E, Erich N, John T.A Method for Transparent Admission Control and Request Scheduling in E-commerce Web Sites[C]. Proceedings of the 13th International Conference on World Wide Web. 2004.

[7]Liu X, Hoe J, Shad L, Zhu X. Adaptive Control of Multi-Tiered Web Application UsinQueuingng Predictor[C]. Proceedings of the 10th IEEE/IFIP Network Operations and Management Symposium,2006.

[8]Urgaonkar B, Shiny P, Chandra A, Goal P, Wood T.Agile dynamic provisioning of multi-tier Internet applications[J]. ACM Trans. on Autonomous and Adaptive Systems,2008,3(1):1-39.

[9]Vilely D, Parham P, Rubenstein D. Provisioning servers in the application tier for e-commerce systems [J]. ACM Trans. on Internet Technology,2007,7(1):1-23. 
[10]Uppsala S, Zhou X P. Coordinated session-based admission control with statistical learning for multi-tier internet applications[J]. Journal of Network and Computer Applications, 2011, 34(1):20-29.

[11] Rio J, Up C.: a coordinated statistical learning approach to measuring the capacity of multi-tier Websites[C]. In Proc. IEEE International Symposium on Parallel and Distributed Processing Sump,2008 